\title{
Spatial filtering of a six-wavelength DBR-RW laser in a MOPA system
}

Tawfieq, M.; Fricke, J.; Stölmacker, C.; Della Casa, P.; Andersen, P. E.; Sumpf, B.; Tränkle, G.

\section{Published in:}

Applied Optics

Link to article, DOI:

10.1364/AO.414883

Publication date:

2021

Document Version

Peer reviewed version

Link back to DTU Orbit

Citation (APA):

Tawfieq, M., Fricke, J., Stölmacker, C., Della Casa, P., Andersen, P. E., Sumpf, B., \& Tränkle, G. (2021). Spatial filtering of a six-wavelength DBR-RW laser in a MOPA system. Applied Optics, 60(7), 1864.

https://doi.org/10.1364/AO.414883

\section{General rights}

Copyright and moral rights for the publications made accessible in the public portal are retained by the authors and/or other copyright owners and it is a condition of accessing publications that users recognise and abide by the legal requirements associated with these rights.

- Users may download and print one copy of any publication from the public portal for the purpose of private study or research.

- You may not further distribute the material or use it for any profit-making activity or commercial gain

- You may freely distribute the URL identifying the publication in the public portal

If you believe that this document breaches copyright please contact us providing details, and we will remove access to the work immediately and investigate your claim 
To be published in Applied Optics:

Title: $\quad$ Spatial filtering of a 6-wavelength DBR-RW laser in a MOPA system

Authors: $\quad$ Mahmoud Tawfieq,Jörg Fricke,Christoph Stölmacker,Pietro Della Casa,Peter Andersen,Bernd Sumpf,Guenther Traenkle

Accepted: 16 January 21

Posted 27 January 21

DOI: $\quad$ https://doi.org/10.1364/AO.414883

(C) 2021 Optical Society of America 


\title{
Spatial filtering of a 6-wavelength DBR-RW laser in a MOPA system
}

\author{
M. TAWfieq,,$^{1,2, *}$ J. Fricke, ${ }^{1}$ C. StölmaCker,${ }^{1}$ P. Della Casa, ${ }^{1}$ P. E. Andersen ${ }^{2}$, \\ B. SUMPF ${ }^{1}$ AND G. TRÄNKLE ${ }^{1}$ \\ ${ }^{1}$ Ferdinand-Braun-Institut, Leibniz-Institut für Höchstfrequenztechnik, 12489 Berlin, Germany. \\ ${ }^{2}$ Technical University of Denmark, Department of Health Technology, Ørsteds Plads, Building 345C, 2800 Kgs. Lyngby, Denmark. \\ *Corresponding author: mahta@dtu.dk
}

Received XX Month XXXX; revised XX Month, XXXX; accepted XX Month XXXX; posted XX Month XXXX (Doc. ID XXXXX); published XX Month XXXX

Lasing emission at multiple wavelengths can be used in different sensing applications and in optical telecommunication. In this work, we report on a six-wavelength distributed Bragg reflector (DBR) laser emitting around $976 \mathrm{~nm}$, where six ridge waveguide (RW) structures with individual DBR gratings are combined into a common front section. These six elements are individually addressable and biased one at a time, for individual wavelength selection. The drawback of this RW combination is seen in the spatial characteristics where higher order modes are supported. This is addressed through a master oscillator power amplifier (MOPA) system which combines the six-wavelength MO laser with a tapered power amplifier. Through this configuration, the PA acts as a spatial filter of the MO beam, providing a nearly diffraction limited beam with $\mathrm{M}_{1 / \mathrm{e}^{2}}<1.5$. In addition, the described MOPA system provides output powers around $4 \mathrm{~W}$ with spectral single mode operation with up to $9.36 \mathrm{~nm}$ of thermal wavelength tuning. We believe that the described MOPA configuration can be used in different applications such as absorption spectroscopy.

OCIS codes: (140.3490) Lasers, distributed-feedback; (140.3600) Lasers, tunable; (250.5980) Semiconductor optical amplifiers; (130.3990) Microoptical devices; (140.3613) Lasers, upconversion.

http://dx.doi.org/10.1364/AO.99.099999

\section{Introduction}

Laser sources that emit at multiple wavelengths can serve in various applications such as optical sensing, absorption spectroscopy, and in sequentially shifted Raman spectroscopy [1-3]. In addition, multiwavelength emission is mostly utilized in wavelength division multiplexing (WDM) systems at $1.55 \mu \mathrm{m}$, where the addition of emission wavelengths is used to increase the transmitted bandwidth through an optical fiber [4]. These and other applications require high output power, excellent beam quality, and spectral single mode operation at multiple wavelengths [4,5].

A prerequisite for multi-wavelength emission is to have a broad gain spectrum and a wavelength tunable selective element. This is e.g. realized through erbium doped fiber (EDF) amplification systems, semiconductor optical amplification (SOA), micro-ring lasers, and fiber Raman amplification (FRA) systems [5-9].

Distributed Bragg reflector (DBR) ridge waveguide (RW) based diode lasers allow single wavelength emission, and in combination with a laser array configuration, multiple wavelengths can be obtained [10]. The drawback is, however, that each laser has its own aperture, and there is a need for spectral beam combining which complicates the system [11].
Alternatively, monolithically combined DBR-RW lasers can provide emission at multiple wavelengths from a single aperture. This is e.g. realized in the so-called Y-branch DBR-RW lasers which provide dualwavelength emission [12]. These light sources are suitable for beat signal generation, shifted excitation Raman spectroscopy, and in terahertz frequency generation [13-15].

The Y-branch lasers provide output powers of between 100 and $250 \mathrm{~mW}$, depending on the emission wavelength and vertical structure $[12,15]$. In order to boost the output power, a master oscillator power amplifier (MOPA) system was previously reported [16]. In such a configuration, the MO provides the wavelength stabilization while the applied tapered power amplifier (TPA) amplifies the output power to the watt range. In addition, we have recently reported on a $23 \mathrm{~nm}$ tunable sampled-grating based MOPA [17].

In this work, we report on a novel six-wavelength DBR-RW laser at wavelength of $975 \mathrm{~nm}$. The introduction of multiple wavelengths and in particular the intersection between the individual RWs, allows higher order spatial modes to propagate [11]. This is compensated through a MOPA configuration, where a TPA is used to filter the spatial characteristics and subsequently provides power amplification. In addition, the described system provides wavelength tunable single mode emission from a nearly diffraction limited beam. 


\section{Setup}

The hybrid MOPA system is sketched in Fig. 1 consisting of a sixwavelength DBR-RW MO laser and a TPA. A hybrid configuration is chosen here to minimize thermal feedback effects between the two chips. As the PA is injected with multiple amperes of current, a high chip temperature is expected for the PA. On the other hand, the MO will be thermally tuned using a micro-heater, which induces a high temperature gradient. Thus, by spacing the MO and PA apart, the thermal coupling is reduced between the two chips.

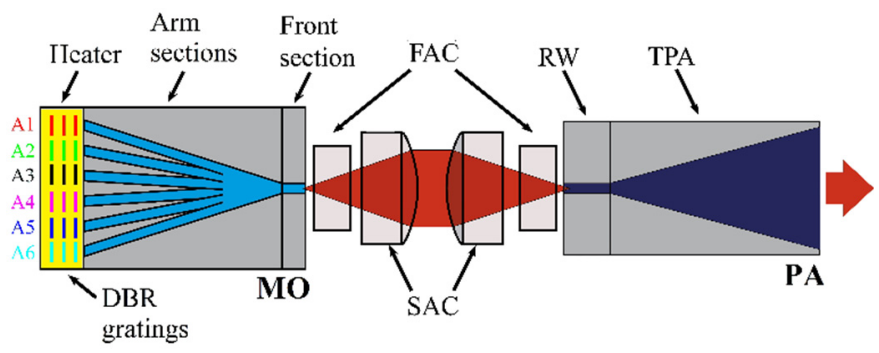

Fig. 1. Sketch of the MOPA system.

The MO has a super-large optical-cavity (SLOC) structure with an InGaAs single quantum well (SQW), sandwiched between AlGaAs waveguide and cladding layers. The exact dimensions as well as the material parameters are described elsewhere [12].

The MO is $4 \mathrm{~mm}$ long, has a RW width of $2.5 \mu \mathrm{m}$, etched down to $\sim 1370 \mathrm{~nm}$ for transverse mode filtering, and has a measured effective index of $n_{\text {eff }}=3.66$. The device consists of three sections; $850 \mu \mathrm{m}$ long DBR grating section, $2600 \mu \mathrm{m}$ long contact section for each arm, and a $500 \mu \mathrm{m}$ long front contact section. Each arm has its own contact for separate current injection.

The front facet of the MO has a 30\% reflectivity coating, while the rear facet has an antireflection (AR) coating with a residual reflectivity of the order of $10^{-4}$. The back facet was AR coated to reduce optical feedback effects of back reflected waves that do not propagate inside the waveguide. The $30 \%$ front facet reflectivity was chosen, as it provided a stable wavelength selection and performance (in comparison with a $5 \%$ reflectivity). This is explained by the reduced coupling between the back reflected light from the PA and into the MO (and the optical elements), as it suppresses a possible cavity arising between the front facets of the PA and the MO.

The wavelength of each arm is defined by individual $7^{\text {th }}$ order deeply etched DBR gratings, which are numbered A1-A6 as shown in Fig. 1. The necessary grating length and residual layer thickness above the quantum well $d$ res was calculated with a simulation tool based on CAMFR (Bienstmann), in order to optimize the reflectivity of the gratings. The calculated reflectivity for $d_{\mathrm{res}}=500 \mathrm{~nm}$ is $90 \%$ for a $1 \mathrm{~mm}$ long grating. Due to the implementation into the waveguide, which is $2.5 \mu \mathrm{m}$ wide and by applying shorter grating lengths, this value is slightly reduced. The gratings pitch is adapted to the different target wavelengths and is around $1.3 \mu \mathrm{m}$. This $7^{\text {th }}$ order grating with a high duty cycle is selected due to manufacturing issues, it is reproducible, and have a low impact on the reflectivity.

In addition, a resistor based micro-heater is implemented on top of the grating section for thermal tuning of each arm. This heater has separate electric contacts from the DBR sections, so that no current is injected into the DBR sections or the gain section below.

The waveguide scheme of the six-arm DBR-RW lasers is plotted in Fig. 2. Each arm consists of two waveguide sections; an angled (dashed) and a bend part (solid), which are combined into the common front section (dotted). The bend sections follow the cosine S-bend formula [18]:

$$
Y(z)=\frac{w}{2}\left[1-\cos \left(\frac{\pi z}{L}\right)\right],
$$

where $Y$ is the lateral position of the waveguide at position $z$, with the offset value of $w$ for a bend length of $L$, see the upper inset of Fig. 2. The half offsets are $w_{\text {inner }}= \pm 40 \mu \mathrm{m}, w_{\text {middle }}= \pm 120 \mu \mathrm{m}$, and $w_{\text {outer }}= \pm 200 \mu \mathrm{m}$ for the inner, middle, and outer arms respectively. The bend length of all three pairs of arms is $L=2000 \mu \mathrm{m}$ defined between $z=[L / 2, L]$. In this range, a single bend is obtained instead of two bends (S-shape) that occurs between $z=[0, L]$, as illustrated in the upper inset of Fig. 2 . The bend losses are reduced by implementing a large bend radius (by setting $w<<L$ ), according to the etch depth and effective index step $[19,20]$. The maximum bending radii of the three arm pairs are $R_{\text {inner }}=$ $28.7 \mathrm{~mm}, R_{\text {middle }}=9.6 \mathrm{~mm}$, and $R_{\text {outer }}=5.8 \mathrm{~mm}$, respectively. These values are estimated as the inverse second derivative [21]:

$$
R \approx\left[\frac{d^{2} Y(z)}{d z^{2}}\right]^{-1} .
$$

The bend sections are connected to RW sections (dashed in Fig. 2), which are angled by $\pm 8.9^{\circ}, \pm 5.4^{\circ}$, and $\pm 1.8^{\circ}$ for the outer, middle, and inner arms, respectively. These values are found to provide a smooth transition between the angled and the bend sections. We have recently reported on a similar waveguide structure (angle + bend) in a Y-branch DBR-RW laser in [12]. The motivation for this waveguide structure is to provide a smooth combination between the individual arms through the bend sections. The angled parts on the other hand, provide an increased spacing between the individual DBR gratings for easier manufacturing and heater implementation. The combination of the 3 individual arm pairs is shown in the lower inset of Fig. 2, indicating the $100 \mu \mathrm{m} z$-axis displacement between the intersection points of the three arm pairs.

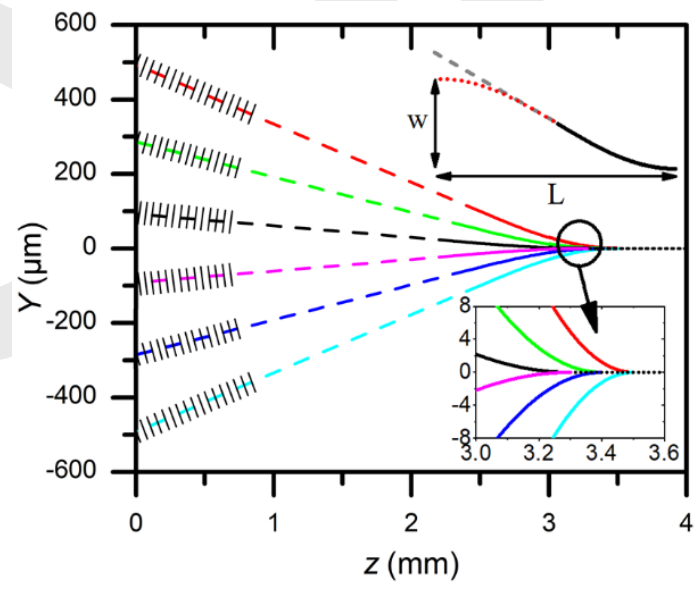

Fig. 2. Sketch of the six-arm DBR-RW laser. The dashed lines represent the angled sections, the solid are the bend sections and the dotted is the common front section. Insets: full S-bend shape and a close-up of the intersection between the three pair of arms.

The PA has an asymmetric SLOC structure with a double quantum well (DQW) active region, guided through AlGaAs waveguide and cladding layers, similar to the one reported in [22]. The PA chip is $6 \mathrm{~mm}$ long and consists of two sections; a $2 \mathrm{~mm}$ long RW ( $5 \mu \mathrm{m}$ wide) followed by a $4 \mathrm{~mm}$ long TPA section with a taper angle of $6^{\circ}$, see Fig. 1 . The two sections of the PA also have individual contacts for separate current injection, which can be used to influence the spatial quality of the tapered structure [23]. The facets of the PA are both AR coated (reflectivity of the order of $10^{-4}$ ), to minimize lasing of the PA itself and to ensure amplification of the MO.

The MO and PA chips are both mounted p-side up on individual CuW sub-mounts, which are then mounted on top of a common AlN heat- 
spreader using AuSn solder. The later serves as the optical bench, which has the dimensions $L \times W \times H=20 \mathrm{~mm} \times 5 \mathrm{~mm} \times 1 \mathrm{~mm}$. Finally, the optical bench is mounted on top of a compact $25 \mathrm{~mm} \times 25 \mathrm{~mm}$ conduction cooled package (CCP).

The vertical and lateral structures of the MO and PA define their individual apertures, which are used to choose a set of fast/slow axis collimation (FAC/SAC) cylindrical micro-lenses, see Fig. 1. The FAC lenses have the dimensions $\mathrm{L} \times \mathrm{W} \times \mathrm{H}=1.5 \mathrm{~mm} \times 0.8 \mathrm{~mm} \times 1 \mathrm{~mm}$, with a focal length of $f=0.6 \mathrm{~mm}$, and a numerical aperture (NA) of 0.8 . The SAC lenses have the dimensions $L \times W \times H=1.5 \mathrm{~mm} \times 1 \mathrm{~mm} \times 1.5 \mathrm{~mm}$, $f=2.3 \mathrm{~mm}$, and $\mathrm{NA}=0.3$. This coupling corresponds to a $1: 1$ imaging of a MO aperture (spot) that is $2.5 \mu \mathrm{m}$ wide and $2 \mu \mathrm{m}$ high, into the PA aperture being $5 \mu \mathrm{m}$ wide and $2 \mu \mathrm{m}$ high.

According to numerical simulations this provides a 0.97 and 0.93 coupling along the horizontal (fast) and vertical (slow) axis, respectively. Note that these simulations only take the fundamental spatial mode and no higher order modes into account. Therefore, it was estimated that only $30 \mathrm{~mW}$ (out of $100 \mathrm{~mW}$ ) is coupled into the PA.

These above numbers were obtained through simulations using the BeamXpertDESIGNER software [24], which uses a ray-transfer (ABCD) matrix method [25]. The lenses are positioned and glued on top of the optical bench using UV-curing adhesive.

The temperature coefficients of the MO and PA are experimentally determined by observing the emission peak wavelength at different (heatsink) temperatures. By assuming a linear dependency between the wavelength and temperature, the MO temperature coefficient is estimated to be $\Delta \lambda / \Delta \mathrm{T}=0.065 \mathrm{~nm} / \mathrm{K}$ while the PA has a coefficient of $0.3 \mathrm{~nm} / \mathrm{K}$. The smaller coefficient of the MO is due to the wavelength stabilization of the DBR grating, while the larger of the PA represents the bulk semiconductor material.

\section{Electro-optical characteristics}

The MO is operated by injecting $I_{\mathrm{MO}}=300 \mathrm{~mA}$ through an arm section and $I_{\text {Front }}=35 \mathrm{~mA}$ into the front section, see Fig. 1 . This provides an estimated coupled $P_{\text {seed }}=30 \mathrm{~mW}$ of seed power into the PA, at a temperature of $15^{\circ} \mathrm{C}$ (temperature kept constant through this study). The low current of $35 \mathrm{~mA}$ is injected into the front section to make this section transparent and non-absorbent. Note that the MO provides more than $100 \mathrm{~mW}$ at the described current setting, but due to the poor beam quality (shown in next section), we estimate that only $30 \mathrm{~mW}$ is coupled into the PA. While the typically required saturation powers of a TPAs lie between 15 and $20 \mathrm{~mW}$ [16], this over-seeding is chosen to provide a uniform output power during the wavelength tuning, as will be shown later.

The output power of the MOPA system is shown in Fig. 3 for $I_{\mathrm{RW}}=$ $300 \mathrm{~mA}$ through the RW section and at different ITPA currents. Comparable maximum output powers $\left(P_{\max }\right)$ are obtained, ranging between 3.6 and $3.9 \mathrm{~W}$ when seeding from the different $\mathrm{MO}$ arms. This indicates that a proper coupling (seeding) is obtained from all six arms of the MO laser.

The emitted wavelengths at $P_{\max }$ are shown in Fig. 4, recorded with a double-echelle monochromator (Demon from LTB Lasertechnik Berlin). This monochromator has a spectral resolution of $17 \mathrm{pm}$ at $975 \mathrm{~nm}$, and was chosen due to its high sensitivity, as the (reflected) wavelength and output power are measured simultaneously. Spectral single-mode operation is observed for all six arms, with full width at half-maximum (FWHM) spectral values limited by the spectrometer. The six emission wavelengths lie between $971.17 \mathrm{~nm}$ and $978.04 \mathrm{~nm}$ with $1.3 \mathrm{~nm}$ wavelength spacing, as intended from the DBR gratings design.

While the described TPA structure can tolerate $I_{\mathrm{TPA}}>15 \mathrm{~A}$ [22], the measurements for this work were stopped at $I_{\mathrm{TPA}}=6 \mathrm{~A}$. This is due to the detuning between the MO wavelengths and the PA gain curve at higher currents, explained by the different temperature coefficients $\Delta \lambda / \Delta T$ of the MO and PA.

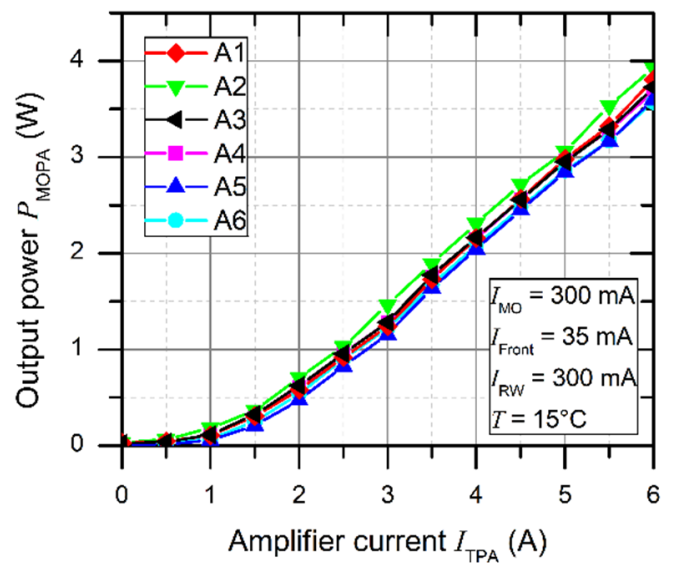

Fig. 3. Output power of the MOPA system at different $I_{\text {TPA }}$ currents.

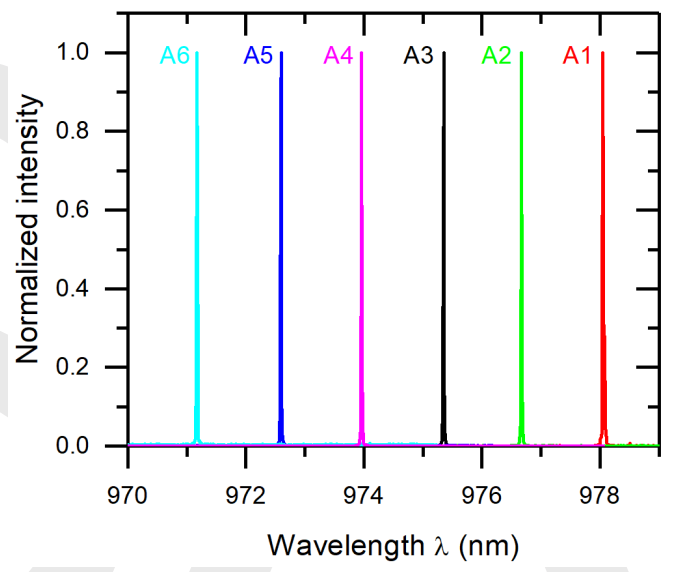

Fig. 4. Emission wavelength of the individual arms at $P_{\max }$.

\section{Spatial characteristics}

\section{A. Beam quality of the MO}

The spatial characteristics of the MO are obtained according to the moving slit method [26]. The normalized lateral intensity distributions of the near fields are shown at the top in Fig. 5 . The beam waist profiles each show a defined central lobe, with a number of low and high intensity side-lobes. The outer arms have the strongest side-lobes, followed by the middle, and the inner arms, respectively. This is likely due to the stronger curvature of the outer arms (smaller bending radii). Symmetry is seen between the side-lobes of the three pairs of arms, indicating that they are caused by the waveguide structures of the individual arms and the intersection of them. The near field widths (measured at $1 / \mathrm{e}^{2}$ ) of the six MO arms are summarized in Table 1. As already noticed, the outer arms have wider beam waists ( 32 and $40 \mu \mathrm{m})$ due to the stronger and wider side-lobes, than the inner four arms (between 20.5-22.0 $\mu \mathrm{m}$ ). These high width values are explained by the RW intersection points between the individual RWs, allowing higher order spatial modes to propagate, although each waveguide is only $2.5 \mu \mathrm{m}$ wide [12].

The far field distributions are likewise obtained and are shown in the bottom in Fig. 5 . A beam steering effect is seen in the far field profiles, as well as multiple symmetrical intensity peaks between the three pairs of 
arms. The far field angles at $1 / \mathrm{e}^{2}$ are shown in Table 1 . The multi-mode and beam steering effects are once again explained by the intersection points between the individual arms, where the light suddenly experiences a RW twice as wide, see the inset of Fig. 2.

By using the measured near field widths and the far field angles, the propagation values $M^{2} 1 / \mathrm{e}^{2}$ in Table 1 are calculated. From these values, it is clear that the outer arms exhibit worse beam quality than the inner four arms, due to the larger bending radii, which is also visible from Fig. 5.

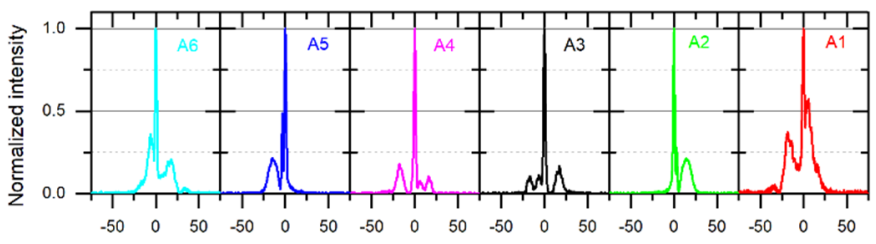
Near field position $(\mu \mathrm{m})$

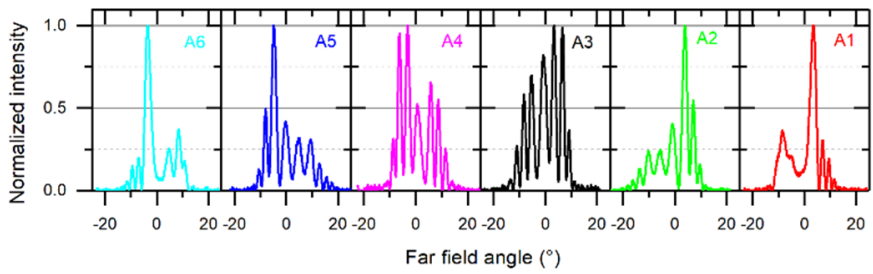

Fig. 5. Near and far fields of the $\mathrm{MO}$ at $I_{\mathrm{MO}}=300 \mathrm{~mA}$ and $I_{\text {Front }}=35 \mathrm{~mA}$

Finally, by considering the near field distributions, the power in the central lobe $P_{\mathrm{CL}}$ of each arm is obtained and summarized in Table 1. Once again, the inner four arms have comparable PCL values between 40 and $50 \%$, while the outer two arms have low values of 26 and $34 \%$. This inconsistency is likely due to the stronger curvature (small bending radii) of the outer two arms which allows the propagation of higher order modes. This being said, all six arms exhibit bad beam qualities, which is the "cost" of combining six individual RWs into a single aperture. At higher $I_{\text {MO }}$ injection currents, the beam quality deteriorates for all six MO arms. However, the overall observed behaviour is maintained, i.e. the large discrepancy between the outer two and four inner arms.

Table 1: Beam quality parameters of the $\mathrm{MO}$ arms at Iмо $_{\text {м }}=300 \mathrm{~mA}$ and for $I_{\text {Front }}=35 \mathrm{~mA}$.

\begin{tabular}{ccccc}
\hline $\begin{array}{c}\text { M0 } \\
\text { arm }\end{array}$ & $\begin{array}{c}\text { Near field } \\
\text { width }[\mu \mathrm{m}]\end{array}$ & $\begin{array}{c}\text { Far field } \\
\text { angle }\left[{ }^{\circ}\right]\end{array}$ & $M^{2} 1 / \mathrm{e}^{2}$ & $\begin{array}{c}P_{\mathrm{CL}} \\
{[\%]}\end{array}$ \\
\hline A1 & 32.0 & 20.8 & 9.4 & 26 \\
A2 & 22.0 & 22.2 & 6.8 & 50 \\
A3 & 21.5 & 21.5 & 6.5 & 51 \\
A4 & 20.5 & 21.5 & 6.2 & 51 \\
A5 & 22.0 & 21.2 & 6.5 & 42 \\
A6 & 41.0 & 20.8 & 12.0 & 34 \\
\hline
\end{tabular}

\section{B. Beam quality of the MOPA}

The corresponding beam waists of the six arms of the MOPA system are shown in Fig. 6. It is here seen that for all six arms, defined central lobes with a number of low intensity side-lobes are obtained. The measured beam waist widths lie between 5 and $6 \mu \mathrm{m}$, except for the case of A1 where a high intensity side lobe leads to a beam waist of $9 \mu \mathrm{m}$, see Table 2. The inset of Fig. 6 shows a slight spread (of maximum $650 \mathrm{~nm}$ ) between the six beam waist peaks. Nevertheless, this measurement indicates that effective coupling should be possible into an $8 \mu \mathrm{m}$ or wider single / low mode fiber.

Likewise, the far field distributions are measured and are shown in Fig. 7. A consistency is seen when comparing the far field distributions, with angle values of about $15.5^{\circ}$. In this measurement, an excellent overlap is observed between the six fields without a sign of beam steering. The corresponding $M^{2} 1 / \mathrm{e}^{2}$ propagation values lie between 1.1 and 1.4, while $\mathrm{A} 1$ has $M^{2} 1 / \mathrm{e}^{2}=2.0$. These values lie typically at $M^{2} 1 / \mathrm{e} 2=1.0$ at low optical powers $\left(I_{\mathrm{TPA}}=3 \mathrm{~A}\right)$ and increases with higher optical powers. Finally, the PCL values are also calculated which show high percentages between 79 and $89 \%$ (70\% for A1). This means that between 2.7 and $3.2 \mathrm{~W}$ of output power lies in the central lobe of the MOPA system. An astigmatism of $1.5 \mathrm{~mm}$ was observed from the MOPA for each arm, indicating once again proper coupling from the individual arms.

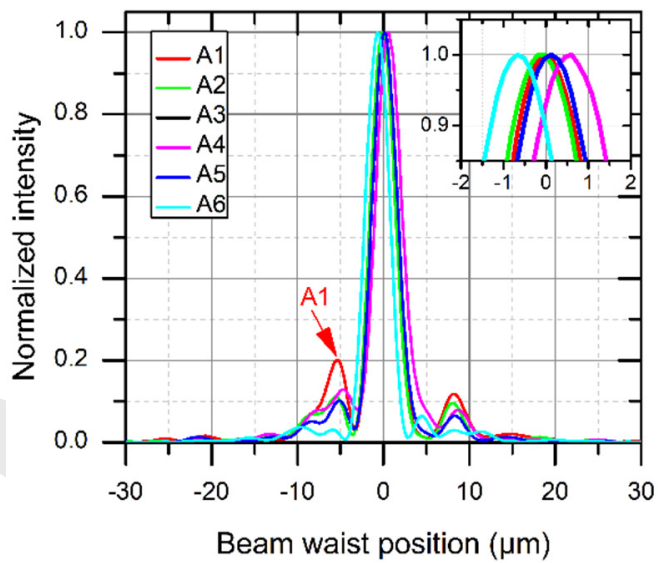

Fig. 6. Normalized beam waist position of the MOPA system at $P_{\max }$. Inset: Zoom of the beam waist peaks.

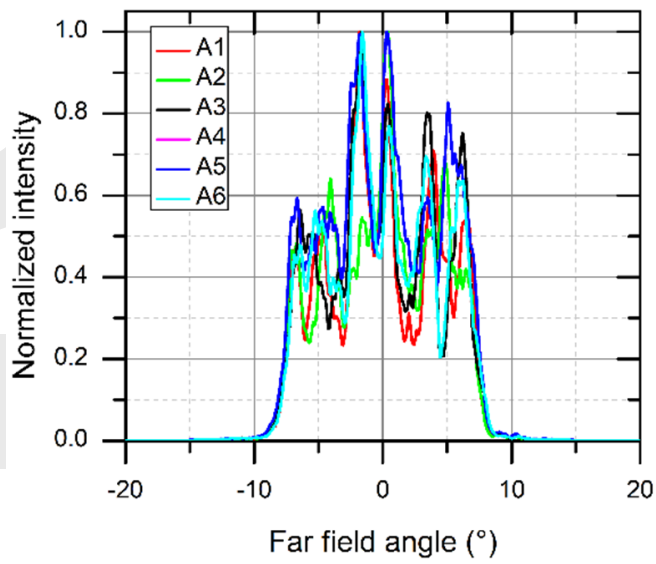

Fig. 7. Normalized far field distribution of the MOPA system at $P_{\max }$.

Table 2: Beam quality parameters of the MOPA at $\boldsymbol{P}_{\max }$ and for the case without seed (PA alone).

\begin{tabular}{ccccc}
\hline $\begin{array}{c}\text { MO } \\
\text { arm }\end{array}$ & $\begin{array}{c}\text { Near field } \\
\text { width }[\mu \mathrm{m}]\end{array}$ & $\begin{array}{c}\text { Far field } \\
\left.\text { angle [ }{ }^{\circ}\right]\end{array}$ & $M^{2} 1 / \mathrm{e}^{2}$ & $\begin{array}{c}P_{\mathrm{CL}} \\
{[\%]}\end{array}$ \\
\hline A1 & 9.5 & 15.3 & 2.0 & 70 \\
A2 & 5.0 & 15.3 & 1.1 & 85 \\
A3 & 5.5 & 15.5 & 1.1 & 77 \\
A4 & 5.5 & 15.5 & 1.2 & 82 \\
A5 & 6.5 & 15.8 & 1.4 & 79 \\
A6 & 5.5 & 15.6 & 1.1 & 89 \\
\hline PA alone & 5.5 & 15.4 & 1.2 & 84 \\
\hline
\end{tabular}


The spatial characteristics of the MOPA system show a clear improvement with nearly diffraction limited beam quality at $P_{\max }$. The PA, in particular its RW section, serves as a spatial filter of the otherwise non-Gaussian beams of the MO. In addition, a more consistent performance is obtained through the MOPA system, both in terms of output power and beam quality, which is necessary in most applications.

For reference purposes, the spatial characteristics of the PA without being seeded are also included (PA alone) in Table 2. These values indicate that the overall beam quality of the MOPA system is in agreement with the PA, and that the system is less influenced by the beam quality of the individual MO arms. For this measurement, the PA was operating in a Fabry-Perot mode, due to the front facet reflectivity (30\%) of the MO.

\section{Wavelength tuning}

As described earlier, a resistor based micro-heater is embedded on top of the grating section of the MO to provide thermal wavelength tuning, see Fig. 1. The measured wavelengths as function of the heater current are combined and shown in a contour plot in Fig. 8. The heater current $I_{\text {heater }}$ was increased up to $400 \mathrm{~mA}$ with a step size of $\Delta I_{\text {heater }}=5 \mathrm{~mA}$.

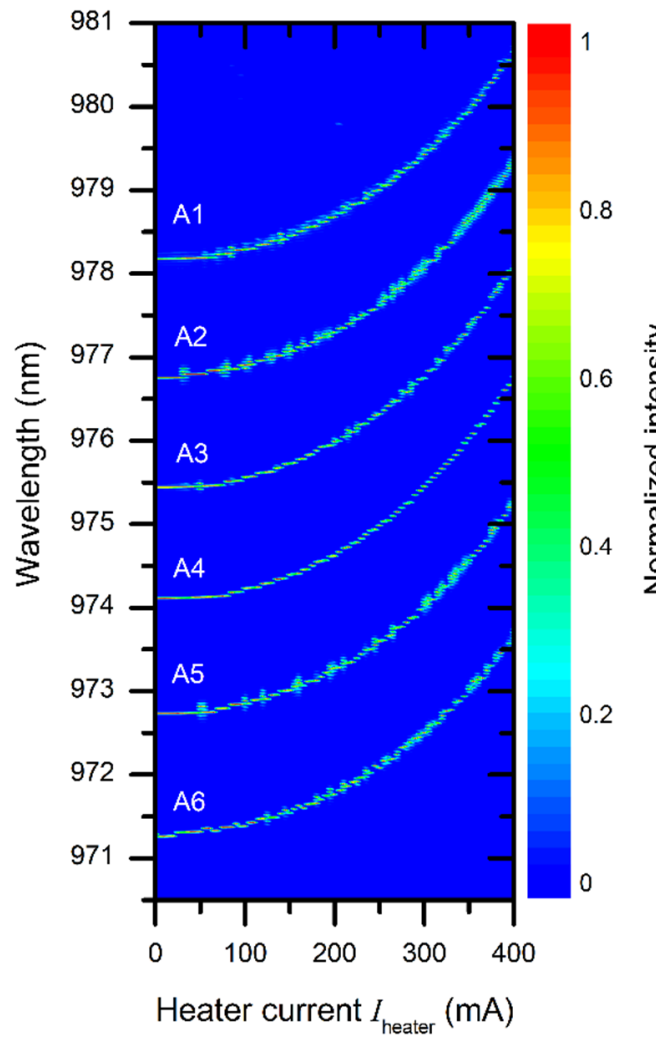

Fig. 8. Combined false colour contour plot of the emission wavelengths of the individual arms at different currents for $P_{\max }$ and at $T=15^{\circ} \mathrm{C}$.

A quadratic tuning behaviour is seen in Fig. 8, due to the Joule heating of a resistor which scales; $P=R \times I_{\text {heater }}{ }^{2}$. The wavelength of each MO arm can be tuned by up to $2.6 \mathrm{~nm}$ as summarized in Table 3 . This indicates that a temperature rise of about $40 \mathrm{~K}$ is induced according to the $\Delta \lambda / \Delta T$ $=0.065 \mathrm{~nm} / \mathrm{K}$ of the MO. With the spectral distance between the individual DBR gratings $(1.3 \mathrm{~nm})$ and with the obtained tuning, a combined $9.36 \mathrm{~nm}$ of tuning range is achieved between 971.29 and $980.65 \mathrm{~nm}$. This being said, these results suggest that by proper placement of each DBR grating, a total tuning of about $2.5 \mathrm{~nm} \times 6=$ $15 \mathrm{~nm}$ can be achieved.

Single mode operation is observed in Fig. 8 from all six arms at lower heater currents, while multi-mode operation becomes more frequentat higher $I_{\text {heater. }}$ This is especially the case in between the individual mode hops, where "multi-mode" operation takes place, with maximum spectral widths of about $262 \mathrm{pm}$ at $1 / \mathrm{e}^{2}$ and around $77 \mathrm{pm}$ at FWHM.

The output power of the MOPA system as function of the heater current is shown in Fig. 9. These measurements indicate small power variations during the wavelength tuning as summarized in Table 3. This low power variation is explained by the over-seeding of the PA. As the temperature of the MO rises with increasing heater currents, the efficiency and thus its output power drops. However, even with this decrease of seed power, the PA is still properly seeded and provides a stable output power.

Due to the limited numerical aperture of the used slow axis collimator, the coupled output power into the power amplifier can vary depended on the position and shape of the far field distribution of the master oscillator. The larger power variation of $4.7 \%$ from arm A5 can be therefore caused by the rather larger far field peak of $-4.8^{\circ}$ (see Fig. 5), in comparison to the values for the other branches. The maximum power for branch 2 on the other side could be due to the unintended adjustment of the lenses to the branch, when manufacturing the device.

Table 3: Wavelength tuning characteristics of the MOPA system.

\begin{tabular}{ccc}
\hline Arm @ $P_{\max }$ & Tuning range $[\mathrm{nm}]$ & Power variation [\%] \\
\hline A1 & 2.48 & 1.1 \\
A2 & 2.57 & 3.3 \\
A3 & 2.62 & 2.2 \\
A4 & 2.65 & 1.2 \\
A5 & 2.53 & 4.7 \\
A6 & 2.38 & 0.6 \\
\hline
\end{tabular}

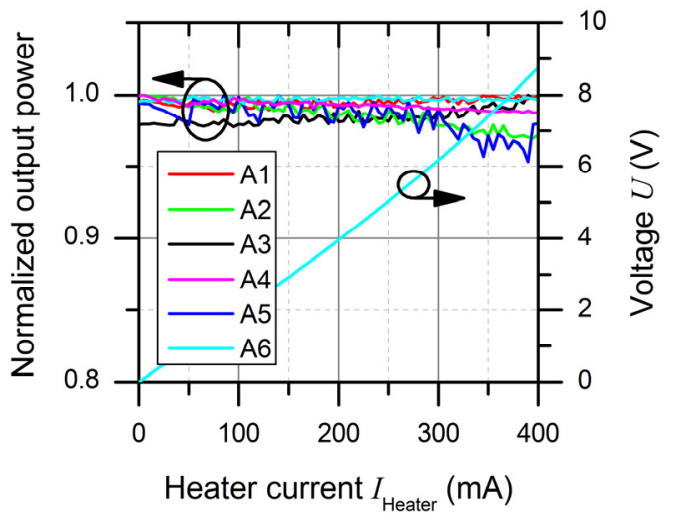

Fig. 9. Normalized output powers of the MOPA (at $P_{\max }$ ) and heater voltage at different heater current $I_{\text {heater }}$ for the six MO arms.

In addition, Fig. 9 shows the heater voltage as function of the applied current. A voltage drop of $U=8.8 \mathrm{~V}$ is measured at a heater current of $I_{\text {heater }}=400 \mathrm{~mA}$. This corresponds to an induced electrical (heater) power of $P=U \times I_{\text {heater }}=3.52 \mathrm{~W}$ and a heater resistance of about $R=$ $U / I_{\text {heater }}=22 \Omega$.

\section{Conclusions}

In this work we report on a compact, tunable, and high power sixwavelength MOPA system. This system utilizes a MO for wavelength stabilization and tuning, while the PA ensures output power in the watt range. The MO is a six-wavelength DBR-RW laser, where six individual 
RWs with corresponding DBR gratings are combined into a common front section. Due to this combination, in particular at the intersection points between the six arms, higher order spatial modes are supported. This is seen in the non-Gaussian beam quality of the six arms with $M^{2} 1 / \mathrm{e}^{2}$ values ranging between 6 and 12 .

By combining the six-arm laser with a TPA, output powers between 3.5 and $4 \mathrm{~W}$ are obtained, with spectral single mode operation (spectral width below $17 \mathrm{pm}$ ). More importantly, the RW section of the PA acts as a spatial filter of the side-lobes of the MO individual arms. This filtering effect is seen as the MOPA system provides a nearly diffraction limited beam, with $M^{2} 1 / \mathrm{e}^{2}$ values between 1.1 and 2.0 and high power in central values between 70 and $90 \%$.

In addition, the wavelength tuning of the MO is demonstrated using a resistor based micro-heater embedded on top of the grating section. Up to $2.6 \mathrm{~nm}$ of wavelength tuning is achieved from the six-arms, which in combination with the gratings spectral spacing provides a total $9.36 \mathrm{~nm}$ of wavelength tuning.

We believe that the described MOPA configuration, together with the observed spatial filtering effect will be useful when considering multiwavelength lasers. In addition, the amplification of the output power together with the wavelength tuning makes this concept ideal for multiple applications. In particular, absorption and spectroscopy applications would benefit from such multi-wavelength lasers.

Funding Information. This work has been carried out under the MidTECH project funded by the European Union's Horizon 2020 research and innovation program under grant agreement no. 642661.

Acknowledgment. The authors gratefully acknowledge J. Mewes for laser measurements, S. Kienast and S. Kreutzmann for laser mounting, P. Ressel for applying the laser coating and D. Feise for providing the power amplifier.

Disclosures. The authors declare no conflicts of interest

\section{References}

1.S. Yamashita, T. Baba, and K. Kashiwagi, "Frequency-Shifted Multiwavelength Fiber Bragg Grating Laser Sensor," Jpn. J. Appl. Phys. 43, $8322-8324$ (2004).

2. J.T.C. Liu, J.B. Jeffries, and R. K. Hanson, "Wavelength modulation absorption spectroscopy with $2 f$ detection using multiplexed diode lasers for rapid temperature measurements in gaseous flows," Appl. Phys. B 78, 503-511 (2004).

3. J. B. Cooper, M. Abdelkader, and K. L. Wise, "Sequentially Shifted Excitation Raman Spectroscopy: Novel Algorithm and Instrumentation for Fluorescence-Free Raman Spectroscopy in Spectral Space," Appl. Spectrosc. 67, 973-984 (2013).

4. S. Sugimoto, K. Minemura, K. Kobayashi, M. Seki, M. Shikada, A. Ueki, T. Yanase, and T. Miki, "High-speed digital-signal transmission experiments by optical wavelength-division multiplexing," Electron. Lett. 13, 22, 680 (1977).

5. A. A. M. Staring, L. H. Spiekman, J. J. M. Binsma, E. J. Jansen, T. van Dongen, P. J. A. Thijs, M. K. Smit, and B. H. Verbeek, "A compact nine-channel multiwavelength laser," IEEE Photonics Technol. Lett. 8, 1139-1141 (1996).

6. M. D. Mermelstein, C. Horn, S. Radic, and C. Headley, "Six-wavelength Raman fibre laser for C- and L-band Raman amplification and dynamic gain flattening," Electron. Lett. 38, 636-638 (2002).

7. C. J.S. D. Matos, D. A. Chestnut, P. C. Reeves-Hall, F. Koch, and J. R. Taylor, "Multi-wavelength, continuous wave fibre Raman ring laser operating at $1.55 \mu \mathrm{m}$," Electron. Lett. 37, 825-826 (2001).

8. M. Khoder, M. Radziunas, V. Tronciu, and G. Verschaffelt, "Study of wavelength switching time in tunable semiconductor micro-ring lasers: experiment and travelling wave description," OSA Continuum 1, 12261240 (2018).

9. X. Dong, P. Shum, N. Q. Ngo, and C. C. Chan, "Multiwavelength Raman fiber laser with a continuously-tunable spacing," Opt. Express 14, 32883293 (2006).

10. O. Brox, J. Fricke, A. Klehr, A. Maaßdorf, M. Matalla, H. Wenzel, and G. Erbert, "24-wavelength distributed Bragg reflector laser array with surface gratings," Electron. Lett. 51, 1352-1354 (2015).

11. T.Y. Fan, "Laser beam combining for high-power, high-radiance sources," IEEE J. Sel. Top. Quantum 11, 567-577 (2005).

12. M. Tawfieq, J. Fricke, A. Müller, P.D.Casa, P. Ressel, A. Ginolas, H. Wenzel, B. Sumpf, and G. Tränkle, "Characterisation and comparison between different S-bend shaped GaAs Y-branch distributed Bragg reflector lasers emitting at 976 nm," Semicond. Sci. Technol. 33, 115001 (2018).

13. R. K. Price, V. B. Verma, K. E. Tobin, V. C. Elarde, and J. J. Coleman, "YBranch Surface-Etched Distributed Bragg Reflector Lasers at $850 \mathrm{~nm}$ for Optical Heterodyning," IEEE Photonics Technol. Lett. 19, 1610-1612 (2007).

14. M. Uemukai, H. Ishida, A. Ito, T. Suhara, H. Kitajima, A. Watanabe, and H. Kan, "Integrated AlGaAs Quantum-Well Ridge-Structure TwoWavelength Distributed Bragg Reflector Laser for Terahertz Wave Generation," Jpn. J. Appl. Phys. 51, 020205 (2012).

15. M. Maiwald, J. Fricke, A. Ginolas, J.Pohl, B. Sumpf, G. Erbert, and G. Tränkle, "Dual-wavelength monolithic Y-branch distributed Bragg reflection diode laser at $671 \mathrm{~nm}$ suitable for shifted excitation Raman difference spectroscopy," Laser Photonics Rev. 7, L30-L33 (2013).

16. M. Tawfieq, A. Müller, J. Fricke, P. D. Casa, P. Ressel, D. Feise, B. Sumpf, and G. Tränkle, "Extended $9.7 \mathrm{~nm}$ tuning range in a MOPA system with a tunable dual grating Y-branch laser," Opt. Lett. 42, 4227-4230 (2017).

17. M. Tawfieq, H. Wenzel, P. Della Casa, O. Brox, A. Ginolas, P. Ressel, D. Feise, A. Knigge, M. Weyers, B. Sumpf, and G. Tränkle, "High power sampled-grating based MOPA system with $23.5 \mathrm{~nm}$ wavelength tuning around 970 nm," Appl. Opt. 57, 8680-8685 (2018)

18. P. L. Liu, B. J. Li, P. J. Cressman, J. R. Debesis, and S. Stoller, "Comparison of measured losses of Ti:LiNbO3 channel waveguide bends," IEEE Photonics Technol. Lett. 3, 755-756 (1991).

19. R. B. Swint, T. S. Yeoh, V.C. Elarde, J. J. Coleman, and M. S. Zediker, "Curved waveguides for spatial mode filters in semiconductor lasers," IEEE Photonics Technol. Lett. 16, 12-14 (2004).

20. R. G. Walker, N. I. Cameron, Y. Zhou, and S. J.Clements, "Optimized Gallium Arsenide Modulators for Advanced Modulation Formats," IEEE J. Sel. Top. Quantum Electron. 19, 138-149 (2013).

21. M. Gray, Modern Differential Geometry of Curves and Surfaces with Mathematica, Second Edition. CRC Press, 1997.

22. C. Fiebig, G. Blume, M. Uebernickel, D. Feise, C. Kaspari, K. Paschke, J. Fricke, H. Wenzel, and G. Erbert, "High-Power DBR-Tapered Laser at $980 \mathrm{~nm}$ for Single-Path Second Harmonic Generation," EEE J. Sel. Top. Quantum Electron. 15, 978-983 (2009).

23. H. Odriozola, J. M. G. Tijero, L. Borruel, I. Esquivias, H. Wenzel, F. Dittmar, K. Paschke, B. Sumpf, and G. Erbert, "Beam Properties of 980$\mathrm{nm}$ Tapered Lasers With Separate Contacts: Experiments and Simulations," IEEE J. Quantum Electron. 45, 42-50 (2009).

24. "BeamXpertDESIGNER." [Online]. Available: https://www.beamxpert.de/, Accessed 26 May 2020.

25. U. Bandelow, and U. Leonhardt, "Light propogation in one-dimensional lossless dielectrica: transfer matrix method and coupled mode theory," Opt. Commun. 101, 1, 92 (1993).

26. "ISO 11146-1:2005(en) Lasers and laser-related equipment-Test methods for laser beam widths, divergence angles and beam propagation ratios-Part 1: Stigmatic and simple astigmatic beams" ISO 11146-1:2005(en). [Online]. Available: https://www.iso.org/obp/ui/\#iso:std:iso:11146:-1:ed-1:v1:en:en/ Accessed 26 May 2020. 